KREBSMEDIZIN HEUTE: Fräventiv, Fersonalisiert, Fräzise und Fartizipativ
32.DEUTSCHER KREBSKONGRESS 2016
CityCube Berlin

24.-27. FEBRUAR 2016

www.dkk2016.de

\title{
Von der Krebsprävention über die Präzisionsmedizin bis zur Patientenbeteiligung
}

Der Deutsche Krebskongress 2016 bietet ein breites Themenspektrum.

Der 32. Deutsche Krebskongress steht bevor vom 24. bis 27. Februar 2016 findet er im CityCube Berlin statt. Erwartet werden mehr als 10.000 Experten, die unter dem Motto „Krebsmedizin heute: präventiv, personalisiert, präzise, partizipativ“ über die jüngsten wissenschaftlichen Ergebnisse der Krebsmedizin diskutieren und dabei auch gesundheitspolitische Aspekte der Krebsversorgung in Deutschland beleuchten. Wie bereits beim letzten Mal wird auch der kommende Krebskongress gemeinsam von der Deutschen Krebsgesellschaft und der Deutschen Krebshilfe ausgerichtet.

\section{Das Motto: Angelehnt an die P4-Medizin}

„Wir haben uns bei der Auswahl unseres Kongressmottos von den Zielen der P4-Medizin nach Leroy Hood leiten lassen", erklärt Prof. Dr. Angelika Eggert, Kongresspräsidentin des DKK 2016. Hood, ein Pionier der biomedizinischen Forschung, entwickelte schon frühzeitig die Vision einer modernen Medizin, die wirksam Krankheiten vorbeugt und molekulare Werkzeuge nutzt, um präzise Diagnosen zu stellen und maßgeschneiderte, auf das individuelle Krankheitsgeschehen abgestimmte Therapien zu entwickeln. Hinzu kommt die aktive Mitwirkung der Patienten, insbesondere bei Therapieentscheidungen auch dieser Punkt kann für den Therapieerfolg wichtig sein. „Diese vier Grundaspekte unseres Mottos ziehen sich wie ein roter Faden durchs Programm des kommenden Kongresses. Allen, die an der Optimierung der Behandlung und Versorgung von Krebspatienten arbeiten, bietet der DKK 2016 eine exzellente Informations- und Diskussionsplattform", betont Prof. Eggert.

\section{Neuer Schwerpunkt:}

Translationale Onkologie

Der Deutsche Krebskongress zeichnet sich durch ein hohes Maß an Interdisziplinarität aus: Von der der onkologischen Chirurgie, der Radioonkologie und medizinischen Onkologie bis hin zur Palliativmedizin und Pflege sind nahezu alle Fachrichtungen vertreten, die bei der Versorgung Krebskranker zusammenwirken. Neu ist dieses Mal der Schwerpunkt „Translationale Onkologie“, der sich mit zahlreichen Sitzungen über alle vier Kongresstage erstreckt.

Weitere thematische Schwerpunkte sind die Supportiv-, Palliativ- und Komplementärmedizin sowie Konzepte für die Betreuung älterer Krebspatienten und Langzeitüberlebender; dazu kommen Plenarsitzungen zur Diagnostik und Therapie verschiedener Krebsarten. Interaktive Tumorkonferenzen und Sitzungen zu aktuellen Leitlinien bieten die Chance, moderne Strategien der Krebsmedizin praxisnah, fächerübergreifend aus der Sicht der Ärzte in Kliniken und Praxen zu diskutieren.

\section{Besondere Angebote für junge Onkologen und Sozialarbeiter}

Der Mangel an jungen Ärzten ist mittlerweile auch in der Onkologie deutlich zu spüren. Speziell für den klinischen Nachwuchs bietet der DKK 2016 vielfältige Möglichkeiten, um sich mit der eigenen wissenschaftlichen Arbeit zu präsentieren. Beim Tag der jungen Medizin können sich junge Ärzte außerdem über Karriereplanung und Fördermöglichkeiten für ihre wissenschaftliche Arbeit informieren. Erstmals beim Kongress vertreten sind auch die onkologisch tätigen Sozialarbeiter mit einem eigenen Programmangebot - die Arbeitsgemeinschaft Soziale Arbeit in der Onkologie (ASO) hat sich 2015 unter dem Dach der Deutschen Krebsgesellschaft gegründet.

\section{Im persönlichen Gespräch:}

Betroffene und Experten

Traditionsgemäß endet der Krebskongress mit dem Krebsaktionstag. Diese Informationsveranstaltung bietet Patienten, Angehörigen und Interessierten die Möglichkeit, sich in

\author{
Vorträgen und im direkten Gespräch mit \\ Experten zu informieren, mit Selbsthilfegrup- \\ pen und Patientenorganisationen Kontakt \\ aufzunehmen und Krebsberatungsangebote \\ kennenzulernen.
}

Alle Informationen, das Vorprogramm und die Onlineanmeldung gibt es auf der offiziellen Webseite www.dkk2016.de.

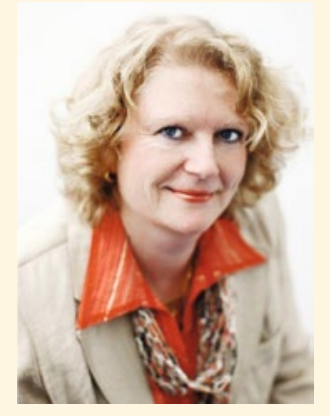

„Beim DKK2016 erwarten Sie über 10.000 Expertinnen und Experten mit ihrem geballten Wissen rund um das Thema Krebs. Die Sichtweisen der Teilnehmer und der interdisziplinäre Diskurs können dem eigenen Denken und Handeln enorme Impulse geben, mit denen man in den beruflichen Alltag zurückkehrt - egal, ob man heilt, forscht oder in der Politik tätig ist. Ich persönlich würde eine solche Gelegenheit nicht missen wollen."

Prof. Dr. Angelika Eggert, Präsidentin des Deutschen Krebskongresses 2016 